\title{
Identification of Critical Parameters Affecting Voltage Stability in Networks with Renewable Generations using Sensitivity Analysis Methods
} DOI:

10.1109/PTC.2017.7981186

\section{Document Version}

Accepted author manuscript

Link to publication record in Manchester Research Explorer

Citation for published version (APA):

Qi, B., \& Milanovic, J. V. (2017). Identification of Critical Parameters Affecting Voltage Stability in Networks with Renewable Generations using Sensitivity Analysis Methods. In PowerTech, 2017 IEEE Manchester https://doi.org/10.1109/PTC.2017.7981186

Published in:

PowerTech, 2017 IEEE Manchester

\section{Citing this paper}

Please note that where the full-text provided on Manchester Research Explorer is the Author Accepted Manuscript or Proof version this may differ from the final Published version. If citing, it is advised that you check and use the publisher's definitive version.

\section{General rights}

Copyright and moral rights for the publications made accessible in the Research Explorer are retained by the authors and/or other copyright owners and it is a condition of accessing publications that users recognise and abide by the legal requirements associated with these rights.

\section{Takedown policy}

If you believe that this document breaches copyright please refer to the University of Manchester's Takedown Procedures [http://man.ac.uk/04Y6Bo] or contact uml.scholarlycommunications@manchester.ac.uk providing relevant details, so we can investigate your claim.

\section{OPEN ACCESS}




\section{Identification of Critical Parameters Affecting Voltage Stability in Networks with Renewable Generations using Sensitivity Analysis Methods}

\author{
Buyang Qi, Student Member, IEEE \\ School of Electrical and Electronic Engineering \\ The University of Manchester \\ Manchester, UK \\ buyang.qi@manchester.ac.uk
}

\author{
Jovica V. Milanovic, Fellow, IEEE \\ School of Electrical and Electronic Engineering \\ The University of Manchester \\ Manchester, UK \\ jovica.milanovic@manchester.ac.uk
}

\begin{abstract}
In modern power systems, the penetration of deregulated market structures, together with the integration of renewable energy source-based generations and nonconventional loads can exhibit inherent stochastic and intermittent behaviour. Hence, bringing uncertainties to the system generation and loading profile, affects the power system's dynamic behaviour. The identification and ranking of critical system uncertain parameters is important for the efficient operation of modern power systems since they can enable better system management with less monitoring. In this paper, 6 sensitivity analysis (SA) methods have been employed for the priority ranking of uncertain parameters in a network with renewable generations from the perspective of their influence on power system voltage stability. The performances of the $6 \mathrm{SA}$ methods are evaluated and their advantages and disadvantages are discussed. The modified version of the 68 bus NETS-NYPS has been used as the test system.
\end{abstract}

Index Terms-Renewable Generation. Sensitivity Analysis. Uncertain Parameters. Voltage Stability.

\section{INTRODUCTION}

Modern power systems have been developed into highly interconnected and complex dynamic systems, and are deeply integrated into society. This makes the secure and stable operation of power systems a significant problem for society as a whole. Several cascading outages took place in the 90's and unveiled the problem of voltage instability within power systems [1-4], and drew the attention of researchers to the area of power system voltage stability analysis. The key characteristics of modern power systems are considered to be a mix of flexible hierarchical control structures, a wide range of electricity generating technologies, and non-conventional loads. These new technologies can make the power systems operate in a more

The authors are with the School of Electrical and Electronic Engineering, The University of Manchester, PO Box 88, Manchester, M60 1QD, UK. (Email: buyang.qi@postgrad.manchester.ac.uk,milanovic@ manchester.a c.uk.). environmentally friendly and flexible manner compared to conventional controlled networks. However, technologies like renewable energy source-based generation and new types of loads can exhibit inherent stochastic behaviour in generations and loading profiles [5]; hence such behaviour is considered a challenge to the steady-state operation of power systems. The number of uncertain parameters within modern power systems can be huge. It is impractical and uneconomical to consider all uncertain parameters and evaluate their impact on system voltage stability behaviour. Fortunately, not all the uncertain parameters contribute the same to system voltage instability, nor do they have the same probability of occurrence. The identification and ranking of influential system parameters based on their impact on system dynamic behaviour can provide an efficient yet accurate approach for power system stability analysis.

This paper employs sensitivity analysis (SA) methods for the identification and ranking of critical uncertain parameters affecting power system voltage stability. Through the ranking of uncertain parameters within a system, components with high impact on system stable operation can be modelled in greater detail and more closely monitored, while others can be treated less intensely to significantly reduce the computational resources required. Previous works have employed 'local' linear algorithms for the identification and ranking of important system parameters such as generators [6], load classification [7], PSS design [8] and PMU placement [9]. More recently an accurate yet computational intensive 'global' approach has been employed in the ranking of frequency support devices [10]. There also exist screening methods like Morris Screening method, which can provide features like reasonable computational cost and acceptable accuracy in one package. The 'screening' method has been 
proved to be effective in systems with a large number of uncertain inputs [11-13].

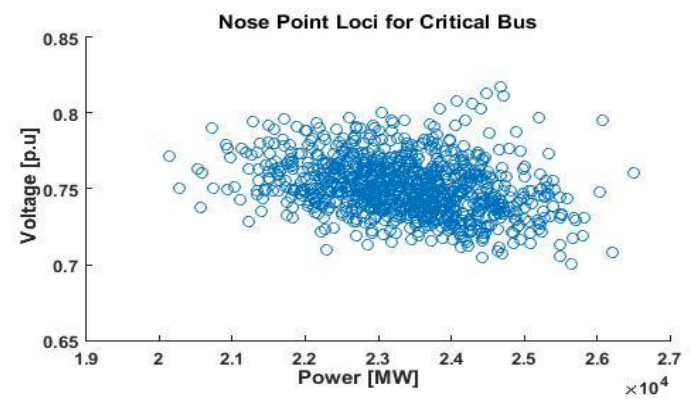

Figure 1 The nose-point locations obtained through different operation conditions

The voltage stability of a power system depends on the relationship between the load bus voltage $(\mathrm{V})$, the reactive power $(\mathrm{Q})$ and the system load consumption $(\mathrm{P})$ [14]. It can be analyzed through P-V curves. In this paper, $\mathrm{P}-\mathrm{V}$ curve plots bus voltage $(\mathrm{V})$ on the system critical bus against total loading $(\mathrm{P})$ of the test network. The test network is stressed under continually increased loading demand until the critical bus experience voltage collapses; hence the nosepoint can be obtained. Past research indicates that with different system operation conditions, the plotted P-V curves and their relative nose-points can be different [15]. Fig. 1 plots 1000 nose-points obtained through simulations with 1000 different system configurations. An important index for voltage stability analysis is the system loadability, or total load (P) of the system [16]. Loadability measures the active power distance between an obtained system nose-point and its corresponding initial operating point. A larger distance indicates that the system can be more loaded before voltage collapse, i.e., the uncertainty of the corresponding parameter is more influential on system voltage stability if leads to wider variation in voltage collapse point.

This paper employs 6 widely-used SA methods for the identification of critical parameters affecting voltage stability of a power system with renewable generations. (i) The One-at-A-Time (OAT) method in the category of local SA method, (ii) the Morris screening method (Morris) in the category of screening SA method, (iii) the Pearson Correlation Coefficient method (PCCE), the Spearman Correlation Coefficient method (SCCE), the Partial Correlation Coefficient method (Partial) and the Sobol Total Indices method (Sobol) in the category of global SA method. These SA methods have been compared against each other, and their corresponding advantages and disadvantages assessed.

\section{Sensitivity Analysis Methods}

Sensitivity Analysis (SA) methods provide an approach for the identification and ranking of critical parameters with uncertainties. It can numerically describe how the variability of input propagates through a modelled system and affects the output result [12].

\section{A. Local SA Method}

Local SA methods evaluate the local impact of one single input parameter on the model output, and are performed by calculating the partial derivatives of the output with respect to the input. One commonly used local SA method is the One-at-A-Time (OAT). This technique changes one input parameter at one time with a small step, only $p+1$ simulations are required for a system with $p$ uncertainties. Local SA methods require very little computational resources; however they suffer from reduced accuracy. They can present unreliable results when the model is nonlinear [13].

\section{B. Global SA Methods}

The global SA methods rank the input parameters by evaluating their effect on model output through the whole set of possible input values. The global methods can be performed on non-parametric uncertainties, for example correlation coefficients, or through the analysis of the output variation like the Sobol indices. The global SA methods can present the most accurate ranking compared to local and screening methods, and hence are always used as benchmarks. But they can be very computational intensive and time consuming $[11,13,17]$. The simulation times required for global SA methods are generally beyond $10^{3}$.

\section{Screening SA Method}

The screening SA methods run multi-dimensional, semi-global search through the range of possible input values and require fewer simulations compared to the global method. The screening methods are considered to be a 'moderate' method since they sacrifice a bit of accuracy for efficiency. The Morris screening method is a commonly-used one since the implementation is relatively straightforward and generally performs better compared to similar ones $[11,13,17,18]$.

There are three important measurements when performing Morris screening method: (1) the elementary effect, (2) the mean value of elementary effect and (3) the standard deviation of the elementary effect.

The elementary effect measures the output variance when an input is changed by a magnitude of $\Delta$.

$$
E E_{p}^{i}(x)=\frac{\left[y\left(x_{1}, x_{2}, \ldots \ldots, x_{i-1}, x_{i}+\Delta, x_{i+1}, \ldots ., x_{p}\right)-y(x)\right]}{\Delta}
$$

In equation (1), $E E_{p}^{i}(x)$ is the elementary effect for inputs, $p$ is the total number of input uncertainties, $\Delta$ is the step that relates to $1 /(r-1)$, and $r$ is the 'level' set for the Morris SA method (usually from 4 to 10).

The mean and standard deviations of the elementary effect are the sensitivity indices for Morris screening method, and they are defined as

$$
\begin{gathered}
\mu_{p}^{*}=\frac{1}{r} \sum_{i=1}^{r}\left|E E_{p}^{i}\right| \\
\sigma_{p}^{*}=\sqrt{\frac{1}{r} \sum_{i=1}^{r}\left(\left|E E_{p}^{i}-\mu_{p}^{*}\right|\right)^{2}}
\end{gathered}
$$

The mean value of the elementary effect measures the sensitivity strength between input and output; an input 
parameter with a larger $\mu_{p}^{*}$ value indicates a higher impact on output, and hence the parameter is identified as

\begin{tabular}{|c|c|c|}
\hline $\begin{array}{c}\text { Load Demand } \\
\text { (Normal distribution) }\end{array}$ & $\begin{array}{c}\text { Wind Generation } \\
\text { (Weibull distribution) }\end{array}$ & $\begin{array}{c}\text { PV Generation } \\
\text { (Beta distribution) }\end{array}$ \\
\hline $3 \sigma=10 \%$ of $\mu$ & $\alpha=2.2, \beta=11.1$ & $\mathrm{a}=13.7, \mathrm{~b}=1.3$ \\
\hline
\end{tabular}

'critical'. The standard deviation of the elementary effect indicates the linearity between input and output. A high $\sigma_{p}^{*}$ means the variable has a non-linear effect on the output and it has interaction with other variables.

The Morris screening method requires $p^{*} r+1$ simulations for a system with $p$ input variables.

\section{TEST SYSTEM CONFIGURATION}

The test network used in this study is a modified version of a reduced order equivalent model of the NETSNYPS (New England Test System-New York Power System) as shown in Fig. 2. There are 16 generators and 68 buses in this system. Two types of renewable energy source-base generations, wind generator and PV generator, are connected to the network. The test network is rated to provide a generation capacity of $17.26 \mathrm{GW}$ (1 p.u). The maximum load demand this network can support before voltage collapses is $1.18 \mathrm{p} . \mathrm{u}$ and the minimum load this network can support without generator disconnection is 0.28 p.u. The detailed data of this network can be found in $[19,20]$.

The uncertain parameters within the test networks are load demand, wind generation and PV generation. These uncertain parameters are probabilistically modelled following Normal Distribution, Weibull Distribution and Beta Distribution, respectively. The model parameters are shown in Table I. There are 49 uncertain parameters in total modelled probabilistically, including load connected at 35 network buses (16 buses with synchronous generators connected to them and 17 buses with no load were excluded) and power output of 14 (7 wind and 7 PV plants) renewable power plants.

The loading scenarios are configured as shown in Table II. It is worth noting that when the system load is reduced, the proportion of renewable generation in the system increases.

The load model chosen for this study is the commonly used exponential load model [21]. And can be expressed as:

$$
\begin{gathered}
P=P_{n}\left(\frac{U}{U_{n}}\right)^{0} \\
Q=Q_{n}\left(\frac{U}{U_{n}}\right)^{2}
\end{gathered}
$$

In the equation, $P$ and $Q$ are the real and reactive power drawn by the load at voltage $U, P_{n}$ and $Q_{n}$ are the real and reactive power drawn under rated voltage $\left(U_{n}\right)$.

The identification and ranking of critical parameters affecting power system voltage stability is conducted through 3 major steps. The probabilistic modelling and sensitivity analysis of input variables are performed in MATLAB. The Optimal Power Flow (OPF) simulation is calculated in MATPOWER. P-V curve analysis is performed in DIgSILENT PowerFactory.

Table I

Probabilistic Distributions and Model Parameters of Uncertain Input Variables of the Test Network

Table II

Loading Scenarios Chosen for the Test Network

\begin{tabular}{|c|c|c|}
\hline Loading Scenarios & System Loading & $\begin{array}{c}\text { Proportion of } \\
\text { Renewables }\end{array}$ \\
\hline High Load & $85 \%(1.00$ p.u $)$ & $12.5 \%$ \\
\hline Low Load & $50 \%(0.57$ p.u $)$ & $25 \%$ \\
\hline
\end{tabular}

IV. RESULTS OF THE ANALYSIS

The six SA methods discussed in Section I have been applied to the test network for the identification of influential parameters.

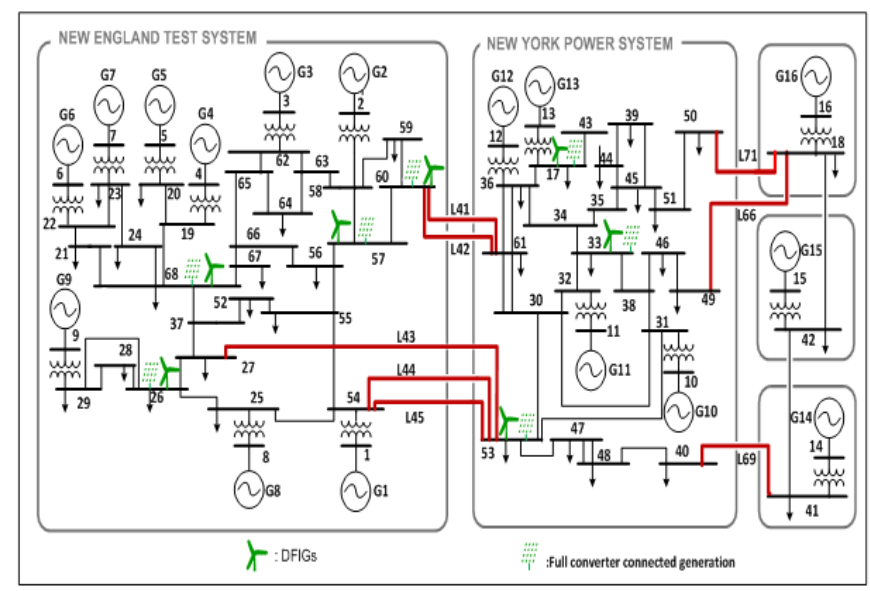

Figure 2 The modified NETS-NYPS test system

\section{A. The Ranking of System Parameters (High Load)}

In Fig. 3, the effect of 35 bus loadings, 7 wind farms and 7 PV farms on system loadability (when the system is stressed under High Load scenario) have been recorded and illustrated. The larger net variation in system loading as a consequence of variation in respective system parameter, the more important parameter is. The abbreviations in front of histograms represent the results obtained from the OAT



Figure 3 Ranking of uncertain parameters through 6 sensitivity analysis methods in High Load Scenario. 
method, Morris screening method, Pearson Correlation Coefficient method, Spearman Correlation Coefficient method, Partial Correlation Coefficient method and Sobol Total Indices method, respectively. Table III

Top 5 Identified System Critical Parameters in High Load Scenario.

\begin{tabular}{|c|c|c|c|c|c|c|}
\hline & OAT & Morris & PCCE & SCCE & Partial & Sobol \\
\hline & \multicolumn{6}{|c|}{ Parameter Name (Parameter No. in Histograms) } \\
\hline \multirow{5}{*}{ 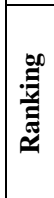 } & L33(12) & L33(12) & L33(12) & L33(12) & L33(12) & L33(12) \\
\hline & L49(23) & W33(41) & W33(41) & W33(41) & W33(41) & W33(41) \\
\hline & L17(1) & W60(36) & S33(47) & S33(47) & $\mathrm{L} 55(28)$ & W53(40) \\
\hline & L55(28) & W26(39) & W53(40) & W53(40) & L60(31) & W60(36) \\
\hline & L60(31) & W53(40) & S53(47) & S53(47) & W53(40) & W17(42) \\
\hline
\end{tabular}

$\mathrm{L}=$ Bus Loading, $\mathrm{W}=$ Wind Farm, $\mathrm{S}=\mathrm{PV}$ Farm, Numbering correspond to system buses in Fig. 2

Table III selects the top 5 identified system critical parameters from 6 different Sensitivity Analysis approaches. These rankings are obtained with respect to the system loadability variation mentioned in Section I. It can be observed that all 6 Sensitivity Analysis methods are capable of identifying the same most influential uncertain parameter, the load variation on bus 33 (No. 12 uncertainty in Fig. 3). However the local search limitation of the OAT method underestimates the importance of load, PV and wind generation connected at bus $36-60$ on system voltage stability behaviour. The ranking obtained through the Morris screening method, though simple to implement and computationally non-expensive, is capable of identifying 4 out of 5 of the same critical parameters as the Sobol Total Indices method. Under this scenario, the renewable generation account for $12.5 \%$ of the generation in the system.
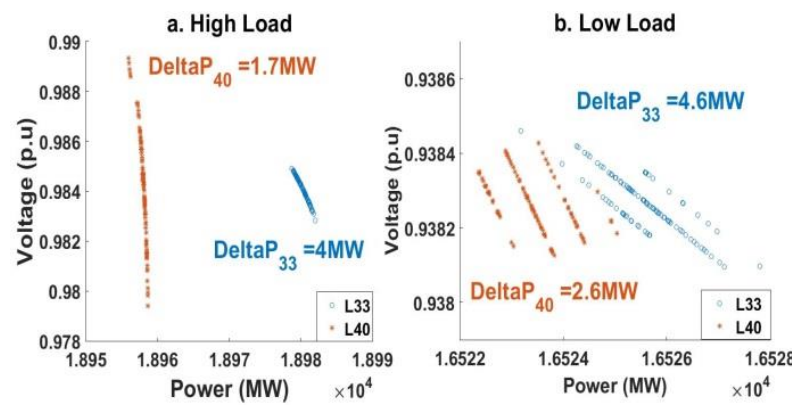

Figure 5 Effect of Critical Parameter vs Less-critical Parameter on voltage collapse point (100 Monte Carlo simulations) under different operation conditions a. High Load (100\% loading $12.2 \%$ penetration of RES) and b. Low Load (57\% loading $21.5 \%$ penetration of RES)

Fig.4 illustrates the variation in critical bus loadability variation when system uncertain parameters with different level of importance are introduced. The most critical parameter L33 (blue) and the less-important parameter L40 (orange) have been chosen for this illustration (both having the same level of uncertainty as defined in Table I). DeltaP is the measure of system loadability variation as a consequence of parameter uncertainty. Fig. 4 indicates that the voltage collapse point is of more sensitive to uncertainty in L33 than to uncertainty in L40.

\section{B. The Ranking of System Parameters (Low Load)}

Fig. 5 illustrates the effects of 49 system input uncertainties on voltage stability calculated through 6 sensitivity analysis methods under low load scenario. The abbreviations in the histograms have the same meaning as those in Fig. 3.

Table IV

Top 5 Identified System Critical Parameters in Low Load Scenario.

\begin{tabular}{|c|c|c|c|c|c|c|}
\hline \multirow{2}{*}{} & OAT & Morris & PCCE & SCCE & Partial & Sobol \\
\cline { 2 - 7 } & \multicolumn{5}{|c|}{ Parameter Name (Parameter No. in Histograms) } \\
\hline \multirow{4}{*}{ 芯 } & L33(12) & L33(12) & W17(42) & W17(42) & L33(12) & W17(42) \\
\cline { 2 - 7 } & W17(42) & W17(42) & S17(49) & S17(49) & W17(42) & L33(12) \\
\cline { 2 - 7 } & S17(49) & W33(41) & L33(12) & L33(12) & W53(40) & W53(40) \\
\cline { 2 - 7 } & W53(40) & S17(49) & W53(40) & W53(40) & W33(41) & W33(41) \\
\cline { 2 - 7 } & W33(41) & S57(44) & S53(47) & S53(47) & S17(49) & W60(36) \\
\hline
\end{tabular}

$\mathrm{L}=$ Bus Loading, W= Wind Farm, S= PV Farm, Numbering correspond to system buses in Fig. 2

In this part of the simulation the test network is operating under low load scenario with system loading demand at $50 \%$ of peak load. Since the system loading demand is reduced compared to high load scenario, the



Figure 4 Ranking of uncertain parameters through 6 sensitivity analysis methods in Low Load Scenario

generation provided by conventional generators is reduced proportionally. The renewable generation now accounts for the $25 \%$ of the generation in the system. In this case 4 out of the 5 sensitivity analysis methods are capable of demonstrating the importance of increased renewable generation penetration on parameter ranking since the wind generation connected to bus 17 is now identified either as the most important or second most important parameter.

From Table IV it can be observed that 3 out of the 4 global sensitivity analysis methods rank the wind generation connected to bus 17 as the most influential input parameter. This time the OAT method is able to identify almost the same parameters selected by global methods, though it still fails to demonstrate the impact of increased proportion of renewable generation due to the limitation of a local search. The result of Morris Screening method again shows good agreement with global methods in identifying the important system parameters. 


\section{Comparison of Sensitivity Analysis Methods}

The considered SA methods are compared from the perspective of simulation times required, accuracy of the ranking results and their complexity of implementation. The test system has 66 uncertain input variables. All the simulations are performed on a PC with Intel ${ }^{\circledR}$ Core $^{\mathrm{TM}}$ i7 processor at $3.4 \mathrm{GHz}$ and $16 \mathrm{~GB}$ of RAM.

The simulation times required for different sensitivity analysis methods depends on the number of model evaluations the simulation performs. For a system with $p$ uncertain input variables, the simulation times required by 6 sensitivity analysis methods are shown in Table V.

In Table $\mathrm{V}, p$ represents the number of system uncertain input, $r$ represents the 'level' of the Morris Screening method (usually from $r=4$ to 10 ), $N$ represents the Number of Monte Carlo Simulation. Table V clearly indicates the huge time and required computational cost differences between local, screening and global sensitivity analysis methods. It is also noted that the simulation times required under low load scenario are longer than those under high load scenario. This is due to the fact that when a system is stressed under low load it is less vulnerable to voltage collapse compared to high load scenario.

Table V

The Number of Simulations and Computational Time Required for Different SA Methods

\begin{tabular}{|c|c|c|c|c|}
\hline $\begin{array}{c}\text { SA } \\
\text { Methods }\end{array}$ & Cost & $\begin{array}{c}\text { No. of } \\
\text { Simulations }\end{array}$ & $\begin{array}{c}\text { Time } \\
\text { (High Load) }\end{array}$ & $\begin{array}{c}\text { Time } \\
\text { (Low Load) }\end{array}$ \\
\hline OAT & $p+1$ & 67 & $28.86 \mathrm{~s}$ & $35.81 \mathrm{~s}$ \\
\hline Morris & $p^{*} r+1$ & $331(r=5)$ & $147.49 \mathrm{~s}$ & $163.01 \mathrm{~s}$ \\
\hline PCCE & $N$ & 1000 & $337.49 \mathrm{~s}$ & $464.23 \mathrm{~s}$ \\
\hline SCCE & $N$ & 1000 & $337.49 \mathrm{~s}$ & $464.23 \mathrm{~s}$ \\
\hline Partial & $N$ & 1000 & $337.49 \mathrm{~s}$ & $464.23 \mathrm{~s}$ \\
\hline Sobol & $(p+1) * N$ & 67000 & $25881.51 \mathrm{~s}$ & $32714.13 \mathrm{~s}$ \\
\hline
\end{tabular}

The Sobol Total Indices method is considered as the benchmark for the evaluation of the accuracy since it has been established in the past that it can be very reliable in case of assessment of non-linear and non-monotonic models $[11,22]$. Table VI presents the accuracy evaluation for 6 sensitivity analyses. The method with a higher correlation coefficient value indicates it can provide a more accurate ranking compared to the Sobol method. Table VI

The Correlation Coefficient Measurement between the Ranking Results from Different SA Methods against Sobol

\begin{tabular}{|c|c|c|}
\hline SA Method & $\begin{array}{c}\text { Correlation Coefficient } \\
\text { against Sobol } \\
\text { (High Load) }\end{array}$ & $\begin{array}{c}\text { Correlation Coefficient } \\
\text { against Sobol } \\
\text { (Low Load) }\end{array}$ \\
\hline OAT & $8.27 \%$ & $13.88 \%$ \\
\hline Morris & $15.54 \%$ & $24.05 \%$ \\
\hline PCCE & $34.52 \%$ & $50.02 \%$ \\
\hline SCCE & $37.61 \%$ & $44.49 \%$ \\
\hline Partial & $50.40 \%$ & $84.59 \%$ \\
\hline Sobol & $100 \%$ & $100 \%$ \\
\hline
\end{tabular}

The presented 6 sensitivity analysis methods can also be compared from the perspective of their effective model complexity level. This has been discussed by previous researchers in $[18,22]$. Morris screening methods and Sobol Total Indices method are both suitable for

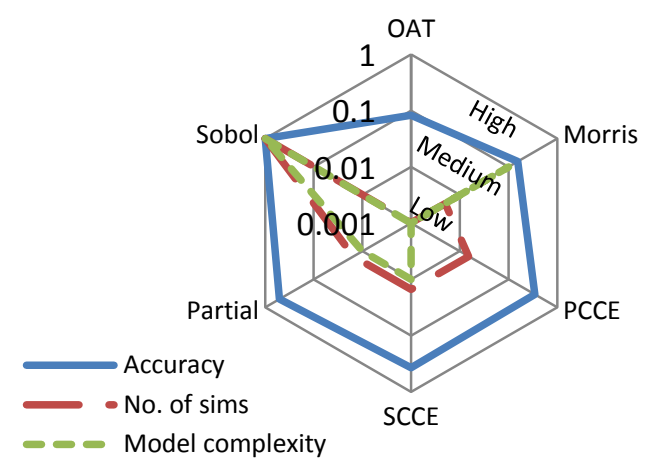

Figure 6 The comparison between 6 SA methods

implementation on Non-monotonic, discontinuous models, which are classified as high-complexity models [18]. The Partial Correlation Coefficient method and Spearman Correlation coefficient method are rank regression-based. They are effective when applied on monotonic models without interactions and considered to be medium complexity methods. One-at-A-Time method and Pearson Correlation Coefficient method are linear regression-based. Their effectiveness is limited to Linear $1^{\text {st }}$ degree models which are of low complexity.

Fig. 6 employs a radar plot to illustrate the comparison between the 6 sensitivity analysis methods from considering the previously discussed criteria.

\section{Discussion}

The rankings of critical system uncertain parameters from Table III and Table IV indicate that the uncertainty in load connected to bus 33 always has a large influence on system voltage stability performance. The contributing factor to this is possibly the fact that the wind and PV plant also connected at bus 33 do not provide any reactive power support when the load at the bus varies. The rankings also highlight uncertainties connected to system bus 53, 60 and 17. Bus 53 and 60 are located near the tielines between New England Test System and New York Power System. Bus 17 is connected to the largest load in the test network. These system topologies contribute to the high scores presented in the ranking results for these uncertainties.

The impact of system loading level can be clearly observed by comparing Fig. 3 and Fig. 5. The decrease in system loading demand is accompanied by an increase in the proportion of renewable generation. The ranking methods then give higher scores to the uncertain parameters related to renewable generations.

Table V and Table VI compare the 6 sensitivity analysis methods based on their corresponding resource consumption and accuracy. Sobol Total Indices method performs best in complex non-parametric models. However it requires too many computational resources and can be unpractical to implement in the system with too many inputs. The OAT method is the quickest approach among the 6 sensitivity analysis methods employed. However it is the least accurate due to its limited local 
search. The Morris screening method stands out from the employed sensitivity analysis methods since it combines efficiency and accuracy. The Morris Screening method can identify 4 out of 5 of the same influential input uncertainties as Correlation Coefficient methods do but it only takes $1 / 3$ of the time. It is also capable of identifying nearly all the influential parameters selected by the Sobol Total Indices method while using only $0.5 \%$ of the computational time required for application of Sobol method.

\section{V.CONCLUSIONS}

The identification and ranking of critical parameters affecting power system voltage stability have been performed in this study through 6 widely-used sensitivity analysis methods. Most of the critical input parameters can be identified by each of the methods.

The 6 employed sensitivity analysis methods can be classified into 3 types. The simplest among all is the Oneat-A-Time method (local SA method). This method is capable of qualitatively identifying the most influential input parameter with a minimal number of simulations. However it fails to correctly rank the input parameters. The Morris screening method (screening SA method) shows a good balance between accuracy and efficiency. The Correlation Coefficient methods (global SA methods) provide more accurate results compared to the previous 2 methods. The Sobol Total Indices method (global SA method) outputs the most accurate ranking of the critical input parameters and consumes the most computational resources.

The comparison between the 6 sensitivity analysis methods based on their application on the test network is performed in Section IV. The poor performance of the local sensitivity analysis method is unfavorable due to the misleading ranking results. The high demand of computational effort from the global sensitivity analysis methods makes them inefficient for many large-scale applications. The Morris screening method employed in this study delivers similar ranking compared to global methods but takes much less time. These properties make the Morris screening method ideal for priority ranking of input uncertainties in large-scale, complex models.

The priority ranking of the influential input parameters based on their impact on power system voltage stability can allocate appropriate monitoring and modelling at selected parameters, and hence increase system security from the perspective of voltage stability. The presented sensitivity analysis methods in this paper can be applied in areas like power system planning and operation for efficient system analyses.

\section{REFERENCE}

[1] C. W. Taylor and D. C. Erickson, "Recording and analyzing the July 2 cascading outage [Western USA power system]," IEEE Computer Applications in Power, vol. 10, pp. 26-30, 1997.
[2] L. Vargas and V. H. Quintana, "Voltage collapse scenario in the Chilean interconnected system," IEEE Transactions on Power Systems, vol. 14, pp. 1415-1421, 1999.

[3] Y. Hain and I. Schweitzer, "Analysis of the power blackout of June 8, 1995 in the Israel Electric Corporation," IEEE transactions on power systems, vol. 12, pp. 1752-1758, 1997.

[4] D. N. Kosterev, C. W. Taylor, and W. A. Mittelstadt, "Model validation for the August 10, 1996 WSCC system outage," IEEE transactions on power systems, vol. 14, pp. 967-979, 1999.

[5] R. Preece, N. C. Woolley, and J. V. Milanović, "The probabilistic collocation method for power-system damping and voltage collapse studies in the presence of uncertainties," IEEE Transactions on Power Systems, vol. 28, pp. 2253-2262, 2013.

[6] F. B. Alhasawi and J. V. Milanovic, "Ranking the importance of synchronous generators for renewable energy integration," IEEE Transactions on Power Systems, vol. 27, pp. 416-423, 2012.

[7] A. M. L. da Silva, J. L. Jardim, L. R. de Lima, and Z. S. Machado, "A Method for Ranking Critical Nodes in Power Networks Including Load Uncertainties," IEEE Transactions on Power Systems, vol. 31, pp. 1341-1349, 2016.

[8] C. Chung, K. Wang, C. Tse, and R. Niu, "Power-system stabilizer (PSS) design by probabilistic sensitivity indexes (PSIs)," IEEE Transactions on Power Systems, vol. 17, pp. 688-693, 2002.

[9] M. Dehghani, B. Shayanfard, and A. R. Khayatian, "PMU ranking based on singular value decomposition of dynamic stability matrix," IEEE Transactions on Power Systems, vol. 28, pp. 2263-2270, 2013.

[10] R. Preece and J. V. Milanović, "Assessing the Applicability of Uncertainty Importance Measures for Power System Studies," IEEE Transactions on Power Systems, vol. 31, pp. 2076-2084, 2016.

[11] A. Saltelli, K. Chan, and E. M. Scott, Sensitivity analysis vol. 1: Wiley New York, 2000.

[12] D. King and B. Perera, "Morris method of sensitivity analysis applied to assess the importance of input variables on urban water supply yield-a case study," Journal of hydrology, vol. 477, pp. 1732, 2013.

[13] K. N. Hasan, R. Preece, and J. V. Milanovic, "Efficient Identification of Critical Parameters Affecting the SmallDisturbance Stability of Power System with Variable Uncertainty," PES General Meeting, 2016.

[14] A. B. Almeida, E. V. De Lorenci, R. C. Leme, A. C. Z. De Souza, B. I. L. Lopes, and K. Lo, "Probabilistic voltage stability assessment considering renewable sources with the help of the PV and QV curves," IET Renewable Power Generation, vol. 7, pp. 521530, 2013.

[15] P. Kundur, N. J. Balu, and M. G. Lauby, Power system stability and control vol. 7: McGraw-hill New York, 1994.

[16] C. W. Taylor, Power system voltage stability: McGraw-Hill, 1994.

[17] X. Song, J. Zhang, C. Zhan, Y. Xuan, M. Ye, and C. Xu, "Global sensitivity analysis in hydrological modeling: Review of concepts, methods, theoretical framework, and applications," Journal of hydrology, vol. 523, pp. 739-757, 2015.

[18] B. Iooss and P. Lemaître, "A review on global sensitivity analysis methods," in Uncertainty Management in Simulation-Optimization of Complex Systems, ed: Springer, 2015, pp. 101-122.

[19] G. Rogers, Power system oscillations: Springer Science \& Business Media, 2012.

[20] B. Pal and B. Chaudhuri, Robust control in power systems: Springer Science \& Business Media, 2006.

[21] K. Yamashita, S. Djokic, J. Matevosyan, F. Resende, L. Korunovic, Z. Dong, et al., "Modelling and Aggregation of Loads in Flexible Power Networks-Scope and Status of the Work of CIGRE WG C4. 605," IFAC Proceedings Volumes, vol. 45, pp. 405-410, 2012.

[22] K. N. Hasan, R. Preece, and J. V. Milanovic, "Priority Ranking of Critical Uncertainties Affecting Small-Disturbance Stability Using Sensitivity Analysis Techniques," IEEE Transactions on Power Systems, 2016. 